\title{
The ACER pollen and charcoal database: a global resource to document vegetation and fire response to abrupt climate changes during the last glacial period
}

\section{F. Sánchez Goñi et al.}

Correspondence to: Maria F. Sanchez Goñi (maria.sanchez-goni@u-bordeaux.fr, maria-fernanda.sanchez-goni@ephe.sorbonne.fr)

The copyright of individual parts of the supplement might differ from the CC BY 3.0 License. 


\section{Supplementary Information}

Taxa defining the pollen percentages of the main biomes in South Africa, Kenya, Australia and New Zealand not included in the QSR special issue (Sánchez Goñi and Harrison, 2010).

\section{Mfabeni Peatland (South Africa)}

Temperate savannah: Anacardiaceae, Ericaceae, Euphorbiaceae, Fabaceae, Fabaceae (Acacia), Proteaceae.

Warm-temperate mixed forest: Apocynaceae, Celastraceae, Combretaceae, Cyanthaceae, Erythroxylaceae, Flacourtiaceae, Moraceae, Myricaceae, Myrtaceae, Podocarpaceae, Rosaceae, Rubiaceae.

\section{Rumuiku Swamp (Kenya)}

Temperate forest : Ilex, Celtis, Lannea, Malvaceae, Rubiaceae, Rhus, Rubus, Stoebe, Merrema, Tiliaceae, Oenostachys, Commelina, Abutilon, Clematis, Cissampelos, Cardamine, Amaranthaceae/Chenopodiaceae, Acanthaceae, Cleome, Cocculus, Plectranthus, Cucurbitaceae, Caryophyllaceae, Cuscuta, Kedrostis, Ranunculus, Gynandropsis, Iridaceae, Hygrophila, Heliotropium, Leucas, Lamiaceae, Liliaceae, Fabaceae, Trema, Valeriana, Ipomoea, Solanum, Urticaceae, Ericaceae, Asteraceae, Brassicaceae, Apiaceae, Artemisia, Poaceae

Warm temperate forest: Dombeya, Myrica, Nuxia, Olea, Moraceae, Podocarpus, Polyscias, Protea, Schefflera, Hagenia, Alchornea, llex, Macaranga, Afrocrania, Celtis, Croton, Juniperus, Rubiaceae, Rapanea, Lasianthus, Syzygium, Capparidaceae, Allophylus, Apodytes, Hypericum, Acalypha, Albizia, Antidesma, Acacia, Bosquea, Canthium, Cliffortia, Neoboutonia, Clausena, Combretum, Clerodendron, Cordia, Drypetes, Dracaena, Phyllanathus, Elatine, Ekebergia, Euclea, Faurea, Gunnera, Gnidia, Ziziphus, Lannea, Malvaceae, Maesa, Phyllanthus, Prunus, Ruelia, Rutaceae, Rubiaceae, Rhus, Rubus, Sapindaceae, Sapotaceae, Tapinanthus, Merremia, Tiliaceae, Oenostachys, Commelina, Abutilon, Clematis, Cissampelos, Cardamine, Amaranthaceae/Chenopodiaceae, Ricinus, Acanthaceae, Cleome, Cocculus, Plectranthus, Cucurbitaceae, Caryophyllaceae, Cuscuta, Chlorophytum, Corchorus, Kohautia, Vernonia, Pavetta, Anthospermum, Ranunculus, Galium, Gynandropsis, Iridaceae, Hyptis, Hygrophila, Leucas, Lamiaceae, Hypoestes, Fabaceae, Trema, Valeriana, Ipomoea, Indigofera, Solanum, Urticaceae, Ericaceae, Asteraceae, Brassicaceae, Apiaceae, Artemisia, Poaceae.

Tropical forest: Dombeya, Myrica, Nuxia, Olea, Moraceae, Podocarpus, Polyscias, Protea, Schefflera, Hagenia, Alchornea, Ilex, Macaranga, Afrocrania, Celtis, Croton, Juniperus, Rubiaceae, Rapanea, Lasianthus, Syzygium, Capparidaceae, Allophylus, Apodytes, Hypericum, Acalypha, Albizia, Antidesma, Acacia, Bosquea, Canthium, Cliffortia, Neoboutonia, Clausena, Combretum, Clerodendron, Cordia, Drypetes, Dracaena, Phyllanathus, Elatine, Ekebergia, Euclea, Faurea, Gunnera, Gnidia, Ziziphus, Lannea, Malvaceae, Maesa, Phyllanthus, Prunus, Ruelia, Rutaceae, Rubiaceae, Rhus, Rubus, Sapindaceae, Sapotaceae, Tapinanthus, Merremia, Tiliaceae, Oenostachys, Commelina, Abutilon, Clematis, Cissampelos, Cardamine, Amaranthaceae/Chenopodiaceae, Ricinus, Acanthaceae, Cleome, 
Cocculus, Plectranthus, Cucurbitaceae, Caryophyllaceae, Cuscuta, Chlorophytum, Corchorus, Kohautia, Vernonia, Pavetta, Anthospermum, Galium, Gynandropsis, Iridaceae, Hyptis, Hygrophila, Lamiaceae, Hypoestes, Fabaceae, Ipomoea, Indigofera, Ericaceae, Asteraceae, Brassicaceae, Apiaceae, Poaceae.

\section{Caledonia Fen and Wagoom (Australia)}

Warm temperate forest: Podocarpus, Phylloclades.

Savannah: Eucalyptus, Casuarina, Poaceae, Asteraceae, Apiaceae, Banksia, Pomaderris, Acacia, Dodonaea, Plantago.

\section{Kohuora (New Zealand)}

Warm temperate forest: Agathis, Alectryon, Ascarina, Dacrydium, Dacrycarpus, Dodonaea, Elaeocarpus, Griselinia, Knightia, Laurelia, Leucopogon fasciculatus, Libocedrus plumosa, Metrosideros, Metrosideros excelesa type, Neomyrtus, Nestegis, Phyllocladus trichomanoides, Plagianthus, Podocarpus, Prumnopitys taxifolia, Prumnopitys ferruginea, Pseudopanax, Weinmannia, Cyathea dealbata type, Cyathea smithii type.

Temperate forest: Fuscospora, Griselinia, Halocarpus bidwillii, Hoheria, Lagarostrobos, Lepidothamnus, Libocedrus bidwillii, Muehlenbeckia, Nothofagus menziesii, Phyllocladus alpinus, Plagianthus, Podocarpus, Quintinia.

Table S1 - List of the applied and selected age models for the sites included in the ACER database.

LI: Liner interpolation; LR: Linear regression; PR2: Polynomial regression-order 2; PR3: Polynomial regression-order 3; PR4: Polynomial regression-order 4; CS: Cubic spline; SSO.3: Smooth spline (smoothing 0.3); SS0.6: Smooth spline (smoothing 0.6); LW0.75: Locally weighted spline (smoothing 0.75). Green cells indicate the selected age model.

No new age model for the following sites: Bear Lake; Lago Grande di Monticchio (too many major inversions in the 14C dates); Okarito Pakihi (lack of dating information); EW9504-17PC; F2-92-P29; ODP 1234; Wonderkrater (Borehole 3); Huiñamarca (Lake Titicaca, lacking dating uncertainties for tephra and $\mathrm{U} / \mathrm{Th}$ dates).

Table S2 - Goodness-of-fit for the selected age models. Green cells indicate the selected age model. Inf: infinite; NA: no available 


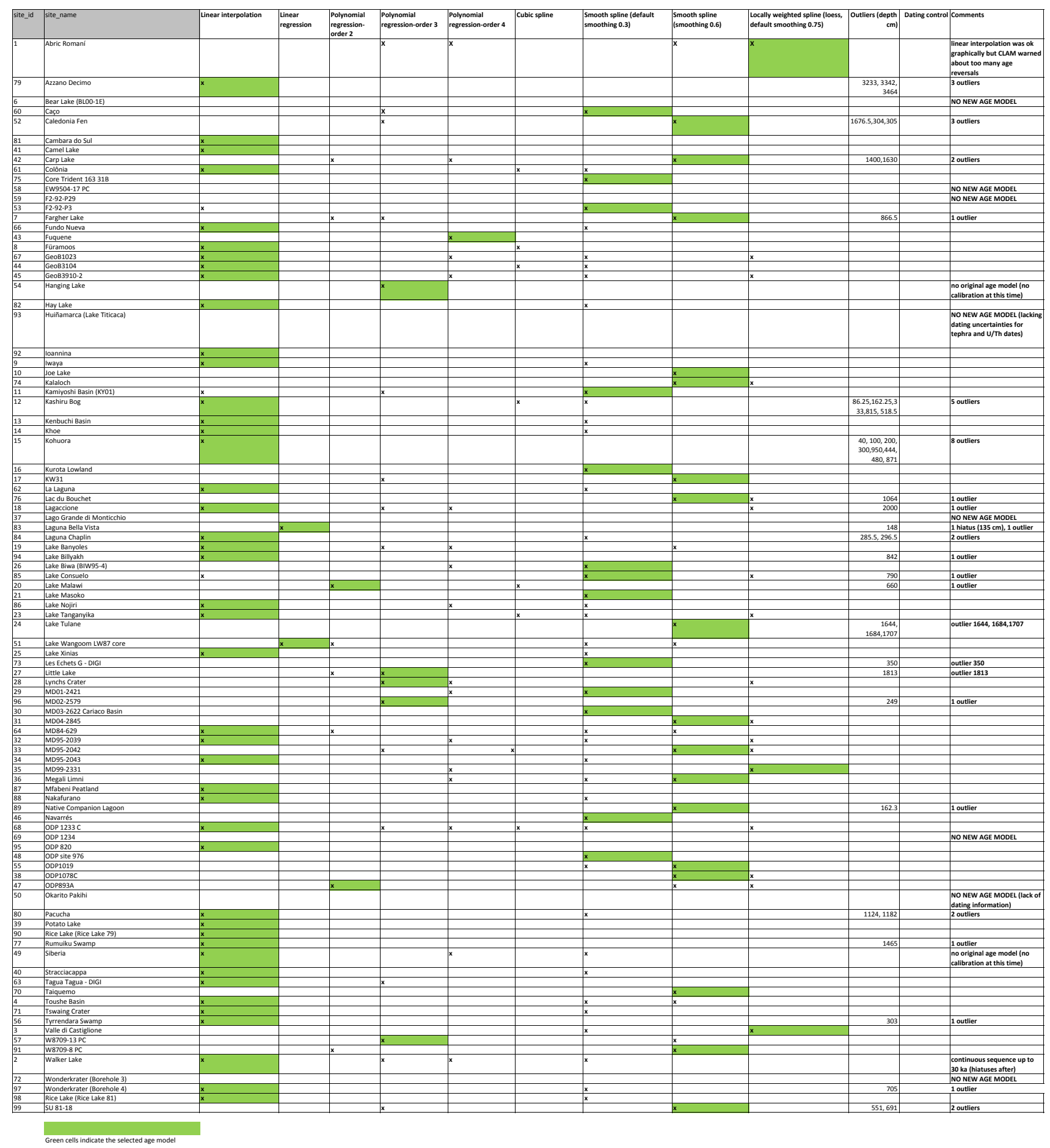




\begin{tabular}{|c|c|c|c|c|c|c|c|c|c|c|}
\hline & A & $\mathrm{B}$ & $\mathrm{C}$ & $\mathrm{D}$ & $E$ & $\mathrm{~F}$ & G & $\mathrm{H}$ & 1 & $\mathrm{~J}$ \\
\hline 1 & sitename & Fit -linear & Fit -reg.lin & \begin{tabular}{|l|} 
Fit -pol.reg2 \\
\end{tabular} & Fit -pol.reg3 & Fit -pol.reg4 & Fit -cubic & Fit-sm.spl0.3 & Fit-sm.spl0.6 & Fit -loess \\
\hline 2 & Abric Romani & NA & 9.7 & \begin{tabular}{|l|}
9.7 \\
\end{tabular} & \begin{tabular}{|l|}
9.7 \\
\end{tabular} & 9.7 & NA & \begin{tabular}{|l|} 
NA \\
\end{tabular} & \begin{tabular}{|l|}
9.7 \\
\end{tabular} & 9.7 \\
\hline 3 & Azzano Decimo & 7.94 & $\operatorname{lnf}$ & NA & NA & NA & NA & NA & NA & NA \\
\hline 4 & Caco & NA & 127.36 & 95.9 & 110.38 & 123.66 & $N A$ & 75.1 & 146.24 & NA \\
\hline 5 & Caledonia Fen & NA & 311.52 & 104.57 & 170.58 & NA & NA & 28.76 & 76.86 & NA \\
\hline 6 & Cambara do Sul & 2 & 226.95 & NA & 2.02 & NA & 2.05 & 6.97 & 175.94 & 2.02 \\
\hline 7 & Camel Lake & 5.03 & 136.84 & 124.25 & NA & $\mathrm{NA}$ & NA & NA & 75.29 & NA \\
\hline 8 & Carp Lake & NA & 109.94 & 62.27 & 55.42 & 50.09 & NA & NA & 53.84 & NA \\
\hline 9 & Colonia & 7.21 & 1002.47 & 437.39 & 113.01 & NA & 7.15 & 20.37 & 574.57 & 43.97 \\
\hline 10 & Core Trident 163 31B & NA & 40.23 & 33.32 & 33.9 & 31.06 & NA & 15.82 & 29.03 & 31.28 \\
\hline 11 & F2-92-P3 & NA & 25.03 & 19.94 & 19.54 & 20.83 & NA & 13.96 & 18.54 & 20.22 \\
\hline 12 & Fargher Lake & NA & 264.77 & 79.58 & 123.18 & NA & NA & NA & 74.12 & NA \\
\hline 13 & Fundo Nueva & 2.72 & 67.9 & 19.7 & 23.07 & NA & NA & 15.08 & 58.39 & NA \\
\hline 14 & Fuquene & NA & 215.7 & 203.56 & 208.83 & 169.95 & NA & NA & 192.66 & NA \\
\hline 15 & Furamoos & 6.99 & 14.99 & 7.25 & NA & NA & 7.28 & NA & NA & 6.94 \\
\hline 16 & GeoB1023 & 3.89 & 44 & 44.22 & 18.68 & 5.72 & 3.97 & 8.59 & 43.54 & 5.84 \\
\hline 17 & GeoB3104 & 5.67 & 35.78 & 32.12 & 40.89 & NA & 5.9 & 14.77 & 31.36 & 23.79 \\
\hline 18 & GeoB3910-2 & 7.03 & 38.78 & 49.75 & 34.15 & 7 & 6.95 & 8 & 39 & 5.06 \\
\hline 19 & Hanging Lake & NA & 233.16 & NA & 60.1 & NA & NA & NA & 58.15 & NA \\
\hline 20 & Hay Lake & 1.81 & 19.88 & 16.43 & 21.75 & NA & $\mathrm{NA}$ & 15.04 & 19.39 & NA \\
\hline 21 & Ioannina & 5.92 & 54.56 & 226.59 & NA & $\mathrm{NA}$ & NA & 73.89 & 83.3 & NA \\
\hline 22 & Iwaya & 2.34 & 19.67 & 27.97 & 2.68 & NA & 2.65 & 6.3 & 19.24 & 2.37 \\
\hline 23 & Joe Lake & NA & 1417.94 & NA & NA & NA & NA & NA & 709.3 & NA \\
\hline 24 & Kalaloch - DIGI & NA & 115.39 & 58.58 & NA & $\mathrm{NA}$ & NA & NA & 18.08 & 19.44 \\
\hline 25 & Kamiyoshi Basin (KY01) & 5.06 & 10.29 & 3.62 & 4.16 & NA & NA & 4.47 & 7.49 & NA \\
\hline 26 & Kashiru Bog & 5.83 & 199.12 & NA & NA & NA & 6.68 & 31.33 & 159.02 & $\mathrm{NA}$ \\
\hline 27 & Kenbuchi Basin & 2.24 & 84.98 & 43.67 & NA & NA & 2.68 & 9.44 & 57.41 & NA \\
\hline 28 & Khoe & 2.45 & 159.64 & 214.92 & NA & NA & NA & 11.95 & 165.05 & NA \\
\hline 29 & Kohuora & 6.05 & $\operatorname{lnf}$ & NA & 144.92 & $\mathrm{NA}$ & NA & NA & 309.92 & $\mathrm{NA}$ \\
\hline 30 & Kurota Lowland & NA & 40.04 & 125.5 & 17.75 & NA & NA & 5.84 & 44.41 & NA \\
\hline 31 & KW31 & $\mathrm{NA}$ & 110.16 & 89.64 & 140.93 & NA & NA & NA & 98.54 & $\mathrm{NA}$ \\
\hline 32 & La Laguna & 3.63 & 320.79 & NA & 117.21 & NA & NA & 56.39 & 233.54 & NA \\
\hline 33 & Lac du Bouchet - DIGI & NA & 24.51 & 24.14 & 25.16 & 23.04 & NA & NA & 19.09 & 17.46 \\
\hline 34 & Lagaccione & 8.35 & 79.33 & 66.41 & 30.7 & 28.2 & NA & NA & 37.4 & 31.82 \\
\hline 35 & Laguna Chaplin & 5.52 & 223.01 & NA & NA & NA & 5.69 & 17.71 & 171.54 & NA \\
\hline 36 & Lake Banyoles & 5.55 & 5.6 & 5.61 & 5.53 & 5.53 & NA & NA & 5.55 & $\mathrm{NA}$ \\
\hline 37 & Lake Billyakh & 6.64 & 1253.57 & 1073.72 & NA & NA & NA & NA & 616.92 & NA \\
\hline 38 & Lake Biwa (BIW95-4) & NA & 55.06 & 37.86 & 19.08 & 20.07 & NA & 16.7 & 32.23 & 22.41 \\
\hline 39 & Lake Consuelo & NA & 291.67 & 25.17 & 23.27 & 25.76 & NA & 15.88 & 42.83 & 23.48 \\
\hline 40 & Lake Malawi & 7.87 & 43.24 & 16.84 & NA & NA & 8.48 & NA & NA & NA \\
\hline 41 & Lake Masoko & NA & 70 & 60.07 & 58.08 & NA & NA & 31.42 & 53.02 & NA \\
\hline 42 & Lake Nojiri & 3.98 & 515.46 & 168.25 & 12.43 & 12.81 & NA & 12.98 & 18.65 & NA \\
\hline 43 & Lake Tanganyika & 4.08 & 40.83 & NA & 7.49 & 5.01 & 3.58 & 4.41 & 20.66 & 3.76 \\
\hline 44 & Lake Tulane & NA & Inf & $\operatorname{lnf}$ & Inf & $\operatorname{lnf}$ & NA & NA & $\operatorname{lnf}$ & NA \\
\hline 45 & Lake Wangoom LW87 core & NA & 12.89 & 12.74 & 5 & 7.53 & NA & 11.34 & 12.74 & NA \\
\hline 46 & Lake Xinias & 4.8 & 82.42 & 17.86 & 5.54 & NA & 5.35 & 11.51 & 68.71 & 5.07 \\
\hline 47 & Les Echets G - DIGI & NA & 70.38 & NA & NA & $\mathrm{NA}$ & NA & 16.61 & 45.19 & NA \\
\hline 48 & Little Lake & NA & Inf & 291.3 & 294.58 & 291.33 & $\mathrm{NA}$ & NA & 269.11 & NA \\
\hline 49 & Lynchs Crater & NA & 381.48 & 214.42 & 177.09 & 182.27 & NA & NA & 178.26 & 168.91 \\
\hline 50 & MD01-2421 & NA & 318.93 & 612.84 & 359.84 & 69.38 & NA & 23.74 & 238.3 & NA \\
\hline 51 & MD02-2579 & NA & 1364.41 & NA & 127.39 & NA & NA & NA & 191.41 & NA \\
\hline 52 & MD03-2622 Cariaco Basin & NA & 11.82 & 11.84 & 11.78 & 11.79 & NA & 11.78 & 11.8 & 11.78 \\
\hline 53 & MD04-2845 & NA & Inf & 52.49 & 28.35 & 29.47 & NA & NA & 22.24 & 26.94 \\
\hline 54 & MD84-629 & 2.77 & 2.91 & 2.9 & 2.77 & NA & 2.77 & 2.82 & 2.91 & 2.77 \\
\hline 55 & MD95-2039 & 10.4 & 10.38 & 10.4 & 10.4 & 10.4 & NA & 10.4 & 10.4 & 10.4 \\
\hline 56 & MD95-2042 & NA & 520.87 & 103.03 & 49.83 & 53.79 & NA & NA & 49.08 & 52.89 \\
\hline 57 & MD95-2043 & 12.73 & Inf & 234.84 & 82.02 & NA & NA & 14.63 & 81.69 & 55.53 \\
\hline 58 & MD99-2331 & NA & 613.51 & 169.98 & 113.75 & 111.31 & NA & NA & NA & 114.26 \\
\hline 59 & Megali Limni & NA & 12.24 & 10.74 & 11.65 & 11.74 & NA & 5.87 & 9.88 & NA \\
\hline 60 & Mfabeni Peatland & 3.08 & 145.52 & 150.1 & NA & NA & NA & 59.33 & 138.83 & 2.92 \\
\hline 61 & Nakafurano & 3.3 & 278.83 & 348.19 & NA & NA & NA & 20.9 & 278.61 & NA \\
\hline 62 & Native Companion Lagoon & NA & 72.74 & 32.09 & 48.52 & NA & NA & NA & 24.87 & NA \\
\hline 63 & Navarres & NA & 548.05 & 615.85 & NA & NA & NA & 47 & 323.64 & 151.78 \\
\hline 64 & ODP $1233 \mathrm{C}$ & 11.56 & 22.5 & 26.55 & 25.91 & 18.58 & NA & 12.59 & 17.4 & 18.45 \\
\hline 65 & ODP 820 & 4.46 & 22.27 & NA & 5.66 & NA & NA & NA & 2.78 & NA \\
\hline 66 & ODP site 976 & NA & 216.2 & 133.71 & 136.6 & 112.46 & NA & 48.06 & 115.98 & 112.16 \\
\hline 67 & ODP1019 & 7.97 & 418.97 & 36.02 & 31.25 & 31.26 & NA & 26.66 & 31.78 & 32 \\
\hline 68 & ODP1078C & NA & 957.42 & 345.83 & NA & NA & NA & NA & 237.79 & 262.05 \\
\hline 69 & ODP893A & NA & 122.22 & 28.51 & 24.11 & 23.68 & NA & 16.86 & 22.07 & 24.27 \\
\hline 70 & Pacucha & 4.91 & 135.24 & 234.58 & 86.2 & 156.76 & NA & 42.77 & 140.32 & NA \\
\hline 71 & Potato Lake & 2.81 & 31.59 & 32.73 & NA & NA & NA & NA & 29.77 & NA \\
\hline 72 & Rice Lake (Rice Lake 79) & 1.57 & 1.58 & NA & NA & NA & 1.58 & NA & NA & NA \\
\hline 73 & Rice Lake (Rice Lake 81) & 3.74 & $\operatorname{lnf}$ & NA & NA & NA & NA & 83.89 & $\operatorname{lnf}$ & NA \\
\hline 74 & Rumuiku Swamp & 5.49 & 440.75 & 275.57 & 128.09 & NA & 6.02 & 66.3 & 434.58 & NA \\
\hline 75 & Siberia & 6.67 & 305.02 & 197.77 & 122.46 & 119.85 & NA & 134.57 & 146.06 & NA \\
\hline 76 & Stracciacappa & 3.27 & 41.6 & 37.87 & 47.99 & 32.3 & NA & 25.56 & 33.46 & 37.96 \\
\hline 77 & SU81-18 & NA & 21.32 & 20.24 & 20.82 & NA & NA & NA & 18.38 & NA \\
\hline 78 & Tagua Tagua - DIGI & 5.18 & 19.39 & 9.82 & 8.92 & 8.02 & NA & NA & 8.03 & 7.74 \\
\hline
\end{tabular}




\begin{tabular}{|c|c|c|c|c|c|c|c|c|c|c|}
\hline & $A$ & B & C & $D$ & $E$ & $F$ & G & $\mathrm{H}$ & I & $\mathrm{J}$ \\
\hline 79 & Taiquemo & NA & 889.16 & 452.44 & 232.04 & 245.97 & $\mathrm{NA}$ & NA & 116.87 & 182.74 \\
\hline 80 & Toushe Basin & 14.55 & 132.02 & 170.44 & 170.5 & 78.18 & NA & 26.45 & 46.22 & 43.61 \\
\hline 81 & Tswaing Crater & 2.08 & 175.16 & 112.94 & NA & NA & NA & 42.41 & 157.45 & 2.13 \\
\hline 82 & Tyrrendara Swamp & 2.46 & 2.56 & 2.34 & NA & NA & 2.35 & NA & NA & 2.5 \\
\hline 83 & Valle di Castiglione & NA & 64.92 & 44.71 & 15.46 & NA & NA & 8.18 & 17.67 & 7.72 \\
\hline 84 & Walker Lake & 6.39 & 25.91 & 19.25 & 9.97 & NA & NA & 8.93 & 19.94 & NA \\
\hline 85 & W8709-13 PC & NA & 94 & 72.59 & 81.3 & 62.6 & $\mathrm{NA}$ & NA & 61.9 & $\mathrm{NA}$ \\
\hline 86 & W8709-8 PC & NA & 410.82 & 381.61 & 277.11 & NA & NA & 48.82 & 178.73 & NA \\
\hline 87 & Wonderkrater (borehole 4) & 13.97 & 513.41 & NA & NA & $\mathrm{NA}$ & 14.38 & 40.7 & 348.38 & $\mathrm{NA}$ \\
\hline 88 & Laguna Bella Vista & NA & 2.88 & NA & $\mathrm{NA}$ & NA & NA & NA & NA & NA \\
\hline
\end{tabular}

